\title{
Prevalence of Achilles Tendinopathy and Associated Selected Intrinsic Risk Factors among Nigerian Footballers
}

\author{
A. I. Aiyegbusi', I. O. Owoeye2, O. J. Balogun³, O. O. Fapojuwo', O. A. Akinloye ${ }^{4}$ \\ 1 Department of Physiotherapy, Faculty of Clinical Sciences, College of Medicine, University of Lagos, Lagos, Nigeria \\ 2 Medical Rehabilitation Therapist Board of Nigeria \\ 3 Department of Biomedical Engineering, Faculty of Basic Medical Sciences, College of Medicine, University of \\ Lagos, Lagos, Nigeria \\ 4 Department of Medical Laboratory Sciences, Faculty of Basic Medical Sciences, College of Medicine, University \\ of Lagos, Lagos, Nigeria
}

\author{
CORRESPONDING AUTHOR: \\ Ayoola Aiyegbusi \\ Department of Physiotherapy \\ Faculty of Clinical Sciences \\ College of Medicine \\ University of Lagos \\ Lagos, Nigeria \\ E-mail: aaiyegbusi@unilag.edu.ng
}

DOI:

10.32098/mltj.01.2021.13

LEVEL OF EVIDENCE: 2B

\begin{abstract}
SUMMARY
Background. Achilles tendinopathy (AT) is a major foot and ankle overuse injury which has been reported to be prevalent in running and jumping sporting activities, especially football which has witnessed increased participation in recent times. However, there are no epidemiological data on tendinopathy in footballers in Nigeria. The aim of this study was to determine the prevalence of Achilles tendinopathy and associated intrinsic risk factors among Nigerian footballers.

Methods. Participants were 151 registered football players recruited from various football clubs in Lagos State, Nigeria. Selected intrinsic factors of age, gender, body mass index (BMI), random blood glucose and bilateral ankle dorsiflexion and plantarflexion were evaluated. AT was determined with the Royal London Hospital Test and ultrasonography. Principal Component Analysis (PCA) was used to determine the association between AT and selected intrinsic risk factors and significance set at $\mathrm{p}<0.05$.

Results. The prevalence of AT was $15.9 \%$. There were significant associations between elevated BMI ( $\mathrm{p}=0.027)$, left ankle dorsiflexion $(\mathrm{p}=0.035)$ and right ankle plantarflexion ( $\mathrm{p}=0.008)$ with Achilles tendinopathy. No significant association ( $\mathrm{p}>0.05)$ was found between Achilles tendinopathy and blood glucose level.

Conclusions. Elevated body mass index, reduced ankle dorsiflexion and plantarflexion are risk factors for Achilles tendinopathy.
\end{abstract}

KEY WORDS

Prevalence; Achilles tendinopathy; intrinsic risk factors; Nigerian footballers.

\section{INTRODUCTION}

Exposure of the Achilles tendon to excessive mechanical loadings during vigorous exercises such as running and jumping result in tendinopathy and subsequent tendon rupture (1). A prevalence of $21 \%$ was reported for Achilles tendinopathy among recreational sports (2) participants in Nigeria (3). The aetiology of Achilles tendinopathy has been reported to be multi-factorial, which could be due to intrinsic and extrinsic factors (4). The intrinsic factors include intra-tendinous degeneration, aging, genetic factors, diabetes, body mass index (BMI) and kinematics and biomechanical abnormalities such as limited range of motion (ROM) and leg length discrepancy $(4,5)$. Some of the extrinsic factors on the other hand are training frequency, trauma, training errors and mechanical overload which all play some role in the development of Achilles tendinopathy (6).

Overweight and obesity as determined by the body mass index (BMI), has been on the increase over the years and it has been reported that both may be associated with poorer fitness in terms of strength and endurance $(7,8)$. This results in lower levels of neuromuscular control including balance and coordination, which could place those with elevated BMI at greater risk for injury (1). A systematic review on running related musculoskeletal injuries found that Achilles tendinopathy presented as the second highest injury incidence at 9.1-10.9\% (7) Saunders et al. (9) reported a 
prevalence of $23.3 \%$ Achilles tendon rupture in footballers among the Danish population in a retrospective study. With increase in football participation in Nigeria, an upsurge in the incidence of injuries has been reported with an overall injury incidence of 113.4 and time-loss incidence of 15.6 injuries/1000 match-hours. Several lower limb injuries such as ankle injuries, anterior cruciate ligament (ACL) ruptures and Achilles tendon injuries have been associated with restricted ankle dorsiflexion range of motion (ROM) (10). A few studies have analyzed whether football-specific adaptations would occur in the ankle and hip rotation ROMs despite the fact that restricted scores have been considered as primary risk factors for some of the most common injuries in football, such as ankle sprains and knee osteoarthritis respectively (11). A study reported that restricted ankle dorsiflexion ROM increases injury risk significantly by modifying lower-limb stiffness and landing forces after a vertical jump (12). It was reported also that the maintenance of blood glucose (energy) balance is an important factor in reduction of injury risk because it ensures optimal performance among athletes (13).

Achilles tendinopathy presents with symptoms of pain, swelling, and impaired function during sporting activities and activities of daily living (4). It is quite detrimental to an athlete's career because it is associated with factors such as psychological discomfort, poor quality of life, time, and productivity loss (14). It has also been associated with functional limitations generally and ankle dorsiflexion has been reportedly reduced as well $(12,15)$.

The quest to identify injury risk factors in sports has been ongoing in the world of sports medicine (10). Currently, there are no documented epidemiological data on Achilles tendinopathy and its associated risk factors among footballers in Nigeria hence he need for this study.

\section{MATERIALS AND METHODS}

This study was a cross sectional analytical survey that involved one hundred and fifty-one (151) amateur football players who were recruited by convenience from various clubs across Lagos, Nigeria. Participants who had played football actively in the last 6 months and currently engaged in full training and match responsibilities at the time of the study were included. Ethical approval was sought and obtained from the institutional Health Research and Ethics Committee and informed consent sought and obtained from the participants after duly explaining the study procedure to them. The consent of the coaches was sought for participants who were under the age of 18 years. This study conformed to the ethical standards of MLTJ (Muscle, Ligaments and Tendons Journal) (16). All anthropometric measurements were taken at the beginning of the study: age, gender, height, and weight of each participant were recorded using the self-administered questionnaire.

Royal London Hospital test was performed on each participant while lying in a prone position on a plinth/flat surface as previously described (17). Participants ware positioned prone on a plinth with their ankles hanging relaxed just over the edge of the plinth, the portion of the Achilles tendon which was maximally tender to palpation was identified. Then the participant was asked to actively dorsiflex the ankle while the tender part of the tendon was palpated again. However, this time in maximal dorsiflexion, participants with Achilles tendinopathy reported a substantial decrease or absence of pain when the palpation technique is repeated in dorsiflexion.

\section{Ultrasonography}

Diagnostic Ultrasound Machine XARIO 200 was used for imaging in gray scale with a $5-12 \mathrm{MHz} 50 \mathrm{~mm}$ linear array transducer as previously described (18). The Achilles tendon of all participants who were positive to the Royal London Hospital test were assessed in prone position with the feet hanging over a plinth. A specialist performed all imaging in the sagittal and axial planes (supero-inferiorly and medio-laterally) along the length of the Achilles tendon. The hypoechoic region and/or focal thickening in both planes were noted. Color Doppler was used to note areas of neovascularization.

\section{Blood Glucose Evaluation}

This was taken in a sitting position, the fingertip of the pollex cleaned with moist cotton wool and lightly pricked with a lancet, a drop of blood was dropped from the pricked region on a test strip and then inserted into the meter. The bleeding area was cleaned with a dry cotton wool. And the sugar level was read off and recorded in millimole per liter $(\mathrm{mmol} / \mathrm{L})$.

\section{Ankle Range of Motion}

The range of motion for plantarflexion and dorsiflexion for each participant was taken by asking the participant to lie supine or sit in a chair and extend their knee on an even/ flat surface. A universal goniometer was placed one degree below the lateral malleolus with the stationary arm parallel to the metatarsals of the foot and the movable arm moved as participants actively flexed and extended their ankles. This measurement was repeated three times and the average score was recorded. All measurements were recorded in degrees. An ROM less than $17^{\circ}$ was regarded as restricted (19). 


\section{Statistical Analysis}

All data was analyzed using descriptive statistics of mean, standard error, percentages, pie-charts, and bar charts. Normality of the data was evaluated using Shapiro-Wilk normality test. Independent student's t-test and Wilcoxon rank-sum test were performed for parametric and non-parametric data to establish statistically significant variables using "ggplot2" and "ggpbur" packages. Principal Component Analysis (PCA) was used to determine the association between Achilles tendinopathy, body mass index and ankle dorsiflexion and plantarflexion. The dataset was randomly partitioned into $80 \%, 20 \%$ for training and testing data. Training data (121 individuals, 6 variables). Testing data (30 individuals, 6 variables). The PCA was conducted on training data. The output of training PCA (selected principal components) was run in logistic regression. The accuracy of logistic regression model in the prediction of Achilles tendinopathy in both training data and testing data were computed and compared. "FactoMineR" and "factoextra R" packages were used to do CPA analysis while "nnet" $R$ package was employed for the analysis of logistic regression. Data was analyzed and plotted using $\mathrm{R}$ statistical computing software version 4.0.2 (20) in RSTUDIO environment. Level of significance for all inferential statistics was set at $\mathrm{p} \leq 0.05$.

\section{RESULTS}

A total of one hundred and fifty-one (151) amateur football players were assessed. One hundred and four (104) which represent $68.9 \%$ of the participants were males and forty-seven (47) $(31.1 \%)$ were females (figure $1 \mathrm{~A}$ ). The Median (Interquartile range) of age (years), height $(\mathrm{m})$, weight $(\mathrm{Kg})$ and $\mathrm{BMI}\left(\mathrm{Kg} / \mathrm{m}^{2}\right)$ are $21(19-25), 1.72(1.65$ 1.78), 63.8 (57-71) and $21.6(19.7-23.3)$ respectively. The characteristics of the participants are seen in table I, while table II shows the distribution of Achilles tendinopathy. The prevalence of Achilles tendinopathy was $15.9 \%$ (figure 1 B). Twenty-four (24) participants tested positive to Royal London Hospital Test while one hundred and twenty-seven (127) were negative. The age distribution of all the participants is described in figure 2. The central tendencies of Age Range of the participants without Achilles Tendinopathy were 18 (17-19), 23 (21-25), 34.3 (0.79) and 42.5 (0.65) while for participants with Achilles Tendinopathy, they were 17.33 (0.56), 23.83 (0.81), 34.44 (1.54) and 42 (0); for Age Range 11-19, 20-29, 30-39 and 40-49, respectively. Where Mdn $(\mathrm{Q} 1-\mathrm{Q} 3)=$ Median (Interquartile range) and $\mathrm{M}(\mathrm{SE})=$ Mean (Standard Error).

Using Wilcoxon rank sum test and independent t-test in the comparison of middle values of participants with and without Achilles tendinopathy. There were significant differences in the body mass index (BMI) $(p=0.005$, figure 3 B), right ankle dorsiflexion (Rom $\mathrm{RD}$ ), right ankle plantarflexion (Rom RP), left ankle dorsiflexion (Rom LD) and left ankle plantarflexion (Rom LP) (all p $<0.05$ ) as seen in figure $4 \mathrm{~A}$, figure $4 \mathrm{~B}$, figure $4 \mathrm{C}$ and figure $4 \mathrm{D}$, respectively. There were no significant differences in Age, Blood Glucose and Parity (all $\mathrm{p}>0.05$ ) as shown in figure $3 \mathrm{~A}$, figure $3 \mathrm{C}$
A

Gender Distribution of the Participants

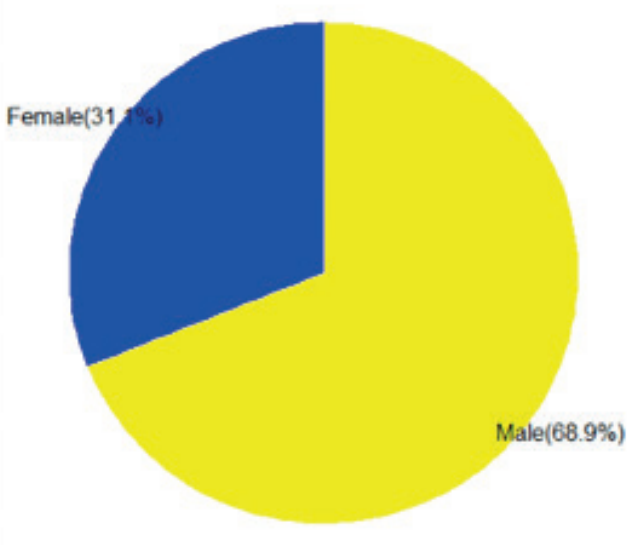

Prevalence of Achilles Tendinopathy (AT)

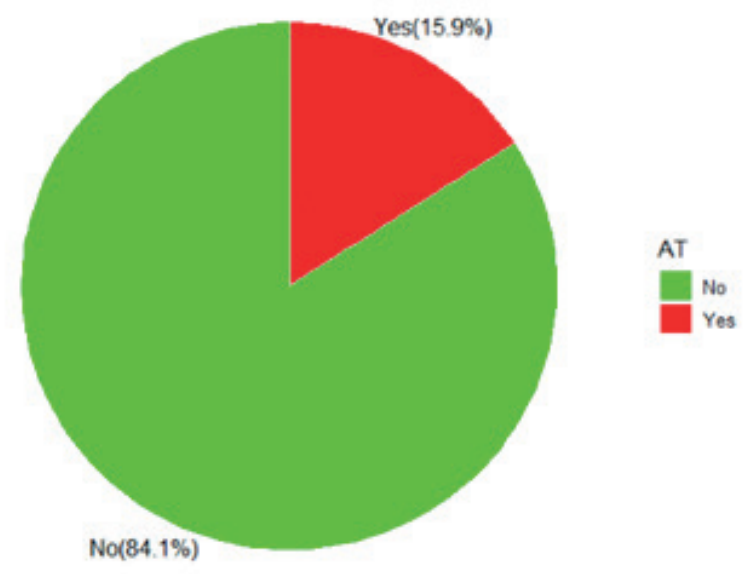

Figure 1. Gender Distribution and Prevalence of Achilles Tendinopathy. 


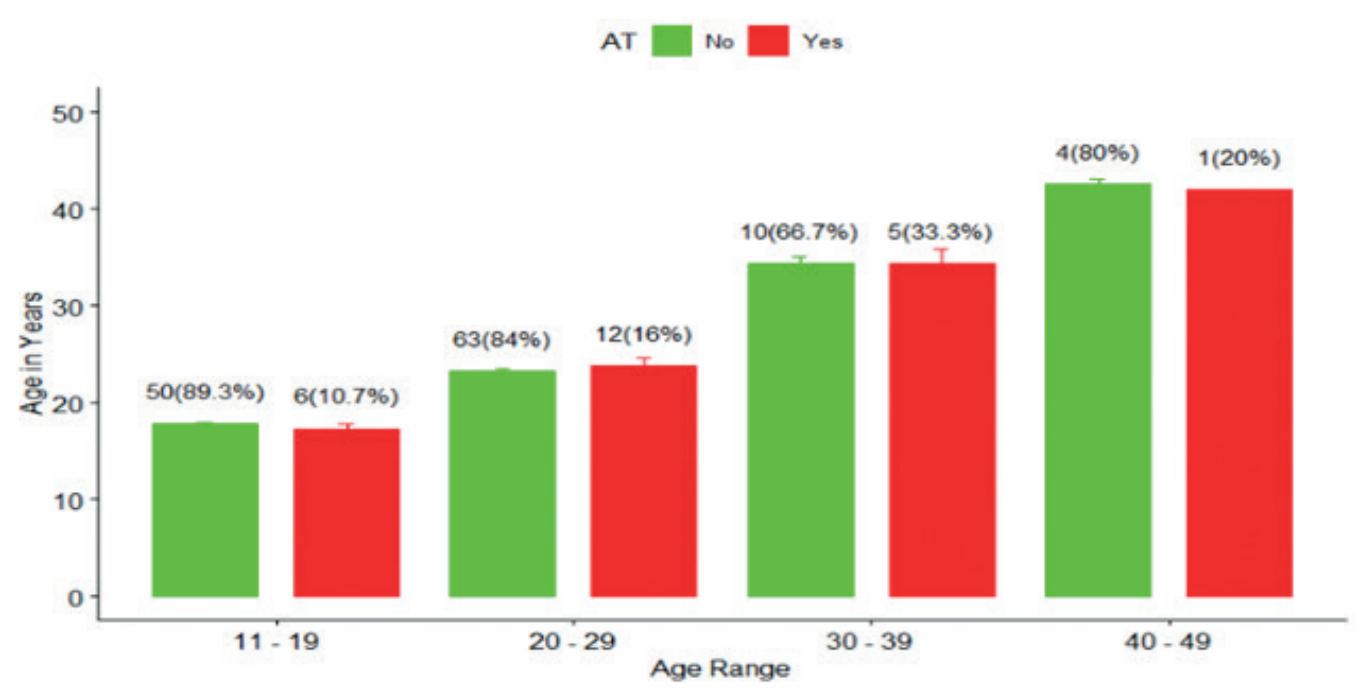

Figure 2. Age distribution of Participants with and without Achilles Tendinopathy.

and figure $3 \mathbf{D}$ accordingly. Principal Component Analysis (PCA) was conducted to see the association of BMI, Rom RD, Rom RP, Rom LD, and Rom LP with Achilles tendinopathy. The principal was used to extract three (3) components. Components with eigenvalues (variances) of less than 1 were not retained for extraction $(20,21)$. The eigenvalue for each principal component were 2.15, 1.19 and 1.07 for PC1, PC2 and PC3 principal component, respectively. The percentage total explained variances by each component were 35.8, 19.8 and 17.8, respectively. The three principal components accounted for $73.4 \%$ of total explained variations (table III). The eigenvectors (rotation matrix) showed

Table I. Characteristics of the Participants.

\begin{tabular}{ll}
\hline Age & $21(19-25)$ \\
\hline Parity & $0(0-0)$ \\
\hline Height $(\mathrm{m})$ & $1.72(1.65-1.78)$ \\
\hline Weight $(\mathrm{Kg})$ & $63.8(57.0-71.0)$ \\
\hline BMI $\left(\mathrm{Kg} / \mathrm{m}^{2}\right)$ & $21.6(19.7-23.3)$ \\
\hline Blood Sugar $(\mathrm{mmol} / \mathrm{L})$ & $4.99(0.06)$ \\
\hline Rom RD $\left(^{\circ}\right)$ & $19(17.5-21)$ \\
\hline Rom RP $\left(^{\circ}\right)$ & $23(20-26)$ \\
\hline Rom LD $\left(^{\circ}\right)$ & $20(19-21)$ \\
\hline Rom LP $\left(^{\circ}\right)$ & $24(21-26)$ \\
\hline
\end{tabular}

ROM - Range of Motion, RD - Right Dorsiflexion, RP - Right Plantarflexion, LD - Left Dorsiflexion, LP - Left Plantarflexion, the values are in Median (Q1-Q3) and Mean (SE).
Table II. Distribution of Achilles Tendinopathy.

\begin{tabular}{|c|c|}
\hline Gender (Male, Female) & $\begin{aligned} 23 & (95.8 \%) \\
1 & (4.2 \%)\end{aligned}$ \\
\hline Type (Mid-Portion, Insertional) & $\begin{array}{l}12(50 \%) \\
12(50 \%)\end{array}$ \\
\hline Affected Leg (Right, Left, Both) & $\begin{array}{c}16(66.7 \%), \\
6(25 \%), \\
2(8.3 \%)\end{array}$ \\
\hline $\begin{array}{l}\text { BMI (Underweight, Normal, } \\
\text { Overweight, Obese) }\end{array}$ & $\begin{array}{c}1(4.2 \%), \\
16(66.7 \%), \\
2(16.7 \%), \\
3(12.5 \%)\end{array}$ \\
\hline $\begin{array}{l}\text { Right Dorsiflexion (Stiffness, Normal, } \\
\text { Hyperflexion) }\end{array}$ & $\begin{array}{c}13(54.2 \%), \\
10(41.7 \%), \\
1(4.2 \%)\end{array}$ \\
\hline $\begin{array}{l}\text { Right Plantarflexion (Stiffness, Normal, } \\
\text { Hyperflexion) }\end{array}$ & $\begin{array}{c}10(41.7 \%), \\
12(50 \%), \\
2(8.3 \%)\end{array}$ \\
\hline $\begin{array}{l}\text { Left Dorsiflexion (Stiffness, Normal, } \\
\text { Hyperflexion) }\end{array}$ & $\begin{array}{c}24(100 \%) \\
0(0 \%) \\
0(0 \%)\end{array}$ \\
\hline $\begin{array}{l}\text { Left Plantarflexion (Stiffness, Normal, } \\
\text { Hyperflexion) }\end{array}$ & $\begin{array}{c}24(100 \%), \\
0(0 \%) \\
0(0 \%)\end{array}$ \\
\hline $\begin{array}{l}\text { Blood Sugar (Hypoglycemic, Normal, } \\
\text { Hyperglycemic) }\end{array}$ & $\begin{array}{c}0(0 \%) \\
24(100 \%), \\
0(0 \%)\end{array}$ \\
\hline
\end{tabular}

ROM - Range of Motion, RD - Right Dorsiflexion, RP - Right Plantarflexion, LD - Left Dorsiflexion, LP - Left Plantarflexion, the values are in Median (Q1-Q3) and Mean (SE). 


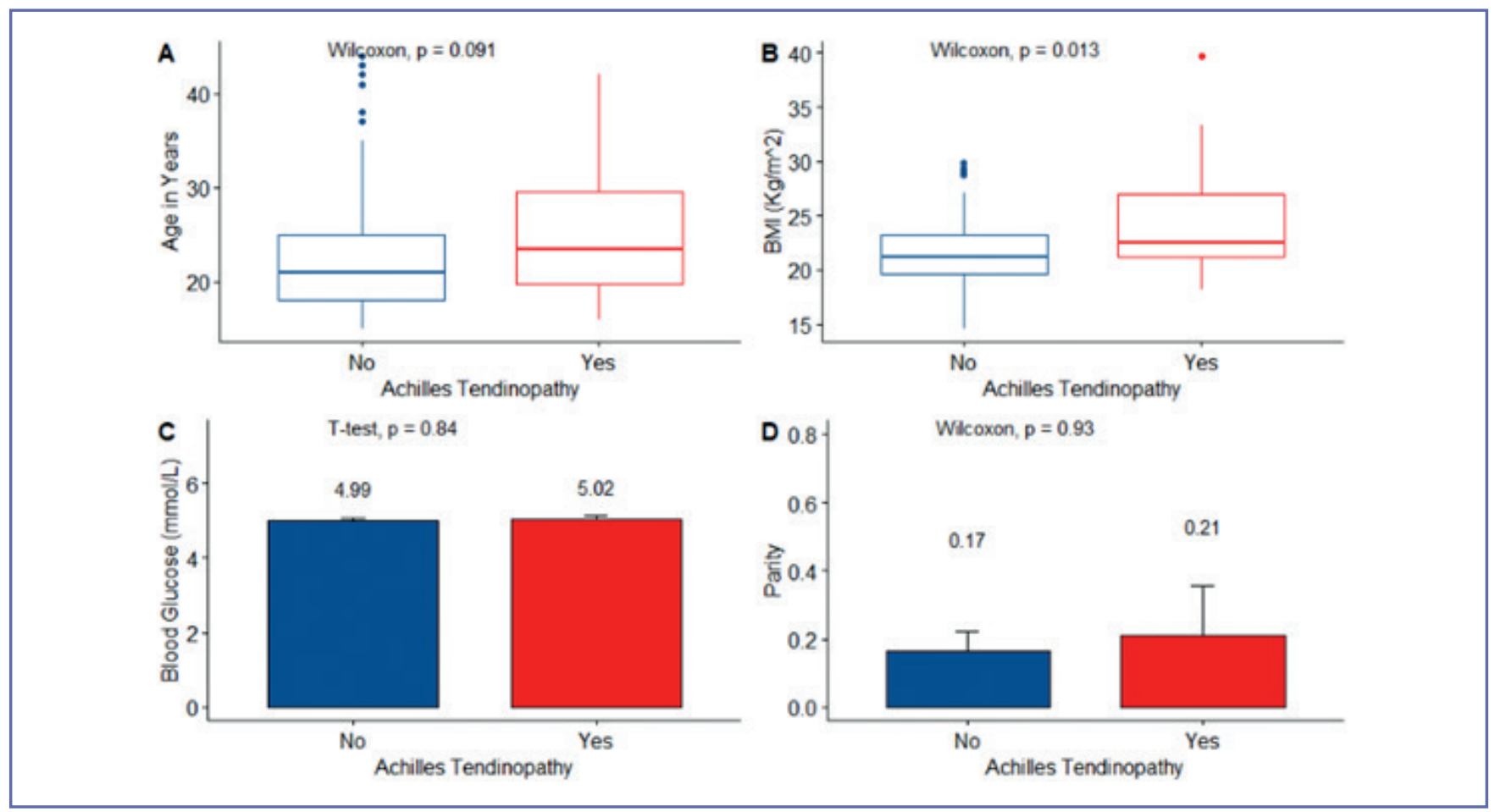

Figure 3. Profiles of Achilles Tendinopathy (ATs) in Age (A), BMI (B), Blood Glucose (C) and Parity (D) of the participants.

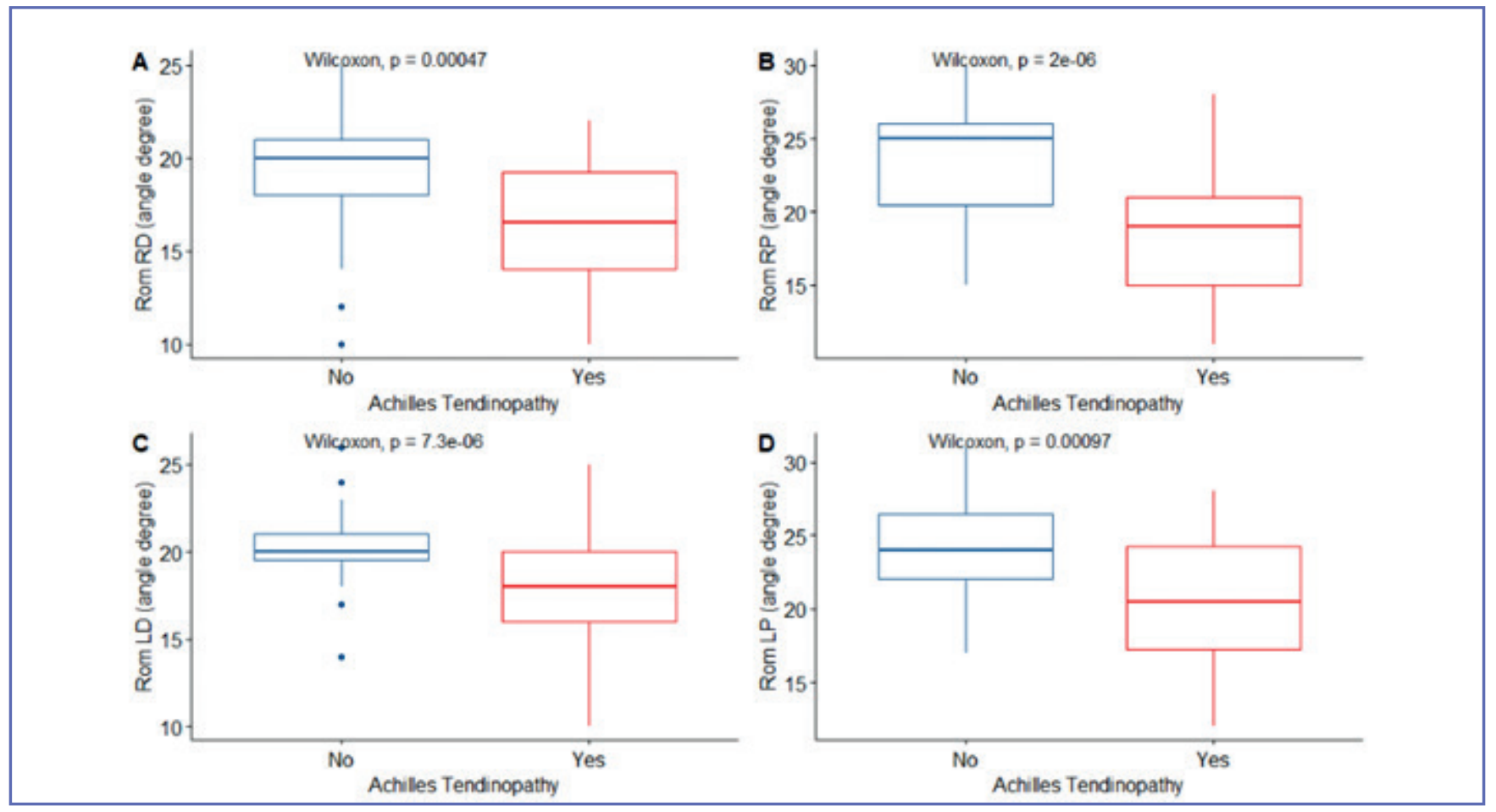

Figure 4. Profiles of Ankle Kinematics. 
Table III. Principal Component Analysis Eigenvalues and Eigenvectors.

\begin{tabular}{lcccccc} 
& PC1 & PC2 & PC3 & PC4 & PC5 & PC6 \\
\hline Normalized Eigenvalues & 2.15 & 1.19 & 1.07 & 0.86 & 0.6 & 0.14 \\
\hline Total Variance Explained (\%) & 35.8 & 19.8 & 17.8 & 14.3 & 10 & 2.3 \\
\hline $\begin{array}{l}\text { Cumulative of Variance } \\
\text { Explained (\%) }\end{array}$ & 35.8 & 55.6 & 73.4 & 87.7 & 97.7 & 1 \\
\hline \multicolumn{7}{l}{} \\
\hline
\end{tabular}

the correlation between the raw variables and transformed variables. Allowing the correlation coefficient $r \geq 0.4$. The first principal component (PC1) explained the greatest proportion of variance and related with four variables of Rom RD, Rom RP, Rom LD, and Rom LP. The second principal component (PC2) is associated with BMI alone. While the third principal component (PC3) is only associated with Blood Glucose Level (table III). Figure $5 \mathbf{A}$ is a two-dimensional biplot of principal component analysis of the participants. The component scores of each individual and the variables loadings were displayed. Although, three principal components were considered in this study, but the first two principal components were plotted for easy illustration and visualization (22). The participants were clustered into group of "Yes" and "No" to Achilles tendinopathy, figure 5 A. Figure $5 \mathbf{B}$ is a factor Map that showed the quality of representation of the variables in PC1 and PC2. Blood Glucose was low in quality, BMI, Rom LD, and Rom LP were moderate and Rom RD and Rom RP were high in quality of representation, figure $5 \mathrm{~B}$. Table IV shows contingency tables (confusion matrices) of logistic regression model of retained principal components. The model accuracies for training and testing data were $87.6 \%$ and $90 \%$ respectively. The difference in the accuracies were not statistically significant $(\mathrm{p}=1.000)$. Also, using logistic regression analysis on the whole dataset, there was a significant association between Achilles tendinopathy and the body mass index (BMI) $\mathrm{p}=0.024$, right plantarflexion $\mathrm{p}=0.008$ and left dorsiflexion $\mathrm{p}=0.038$ (table $\mathrm{V}$ ). As regards the random blood glucose, one hundred and forty-five $(97.9 \%)$ of the participants had normal blood glucose level (random and after meal), two (1.4\%) had low blood glucose level and one $(0.7 \%)$ participant was hyperglycemic. There was no signif- icant association between Achilles tendinopathy and blood glucose level of the football players $(\mathrm{p}=0.7464)$ as all the participants with AT had normal glucose levels. However, the third principal component (PC3) is only associated with Blood Glucose Level (table III).

\section{DISCUSSION}

Epidemiological evidence of tendon pathology among sportspersons in Nigeria is very limited even though the importance of this knowledge, coupled with identifying associated risk factors cannot be over emphasized. This was a cross-sectional study to determine the prevalence of Achilles tendinopathy and its association with some selected intrinsic risk factors among Nigerian footballers. This knowledge is pertinent even as a prior study had reported the dominance of intrinsic factors over extrinsic factors in the aetiology of tendinopathy (23). There is presently no epidemiological data on tendinopathy among footballers in Nigeria, this study has therefore provided preliminary data as well as identified intrinsic factors which will enable preventive measures to mitigate their effects on tendons.

The prevalence of Achilles tendinopathy (AT) in this study was $15.9 \%$ while the findings by Docking et al. (15) reported a prevalence of $21.5 \%$ among Australian male professional football players. The prevalence of AT in this study was lower than what was reported in a similar study on recreational athletes in Nigeria (1). The lower prevalence reported in this study could be due to the relatively small sample size, thus studies with much larger sample sizes are advocated. Our findings also showed that half $(50 \%)$ of the footballers had midportion AT while the other half presented with the insertional type (table II), thus both were equally prevalent. 
Table IV. Confusion Matrix of PCA Performance.

\begin{tabular}{|c|c|c|c|c|c|}
\hline \multirow[t]{2}{*}{$\begin{array}{l}\text { PCA Model } \\
\text { Prediction } \\
\end{array}$} & \multicolumn{2}{|c|}{ Training Data } & \multirow[t]{2}{*}{$\begin{array}{l}\text { PCA Model } \\
\text { Prediction }\end{array}$} & \multicolumn{2}{|c|}{ Testing Data } \\
\hline & No & Yes & & No & Yes \\
\hline No & 97 & 12 & No & 25 & 1 \\
\hline Yes & 3 & 9 & Yes & 2 & 2 \\
\hline \multicolumn{3}{|c|}{ Accuracy $=87.6 \%(95 \%$ CI; $80.38 \%-92.89 \%)$} & \multicolumn{3}{|c|}{ Accuracy $=90 \%(95 \% \mathrm{CI} ; 73.47 \%-97.89 \%)$} \\
\hline \multicolumn{3}{|l|}{ Error rate $=12.4 \%$} & \multicolumn{3}{|l|}{ Error rate $=10 \%$} \\
\hline \multicolumn{6}{|c|}{ Fisher' s Exact, p value $=1$, Odds ratio $=0.79(95 \%$ CI; 0.14-3.08) } \\
\hline
\end{tabular}

Our results showed that $96 \%$ of the participants with AT were males while only one was female. This was not unexpected as the hormone estrogen has been documented to protect pre-menopausal women from central fat accumulation which is one of the symptoms of metabolic syndrome (24). A prior study had reported metabolic syndrome as a risk factor for Achilles tendon pathology in men because men usually have less total body fat but more central/intra-abdominal adipose tissue, whereas women have more total fat mainly in the gluteal/femoral and subcutaneous depots with a reduction in central adiposity. The reduced central adiposity in women therefore may serve as a preventive factor against tendon damage in pre-menopausal women (25). As seen in the age distribution of the participants in this study, all the female footballers are at the pre-menopausal stage.

\section{The epidemiology of tendon injuries}

Ruptures and tendinopathy have highlighted the association between BMI or adiposity and the risk of pathology (26).

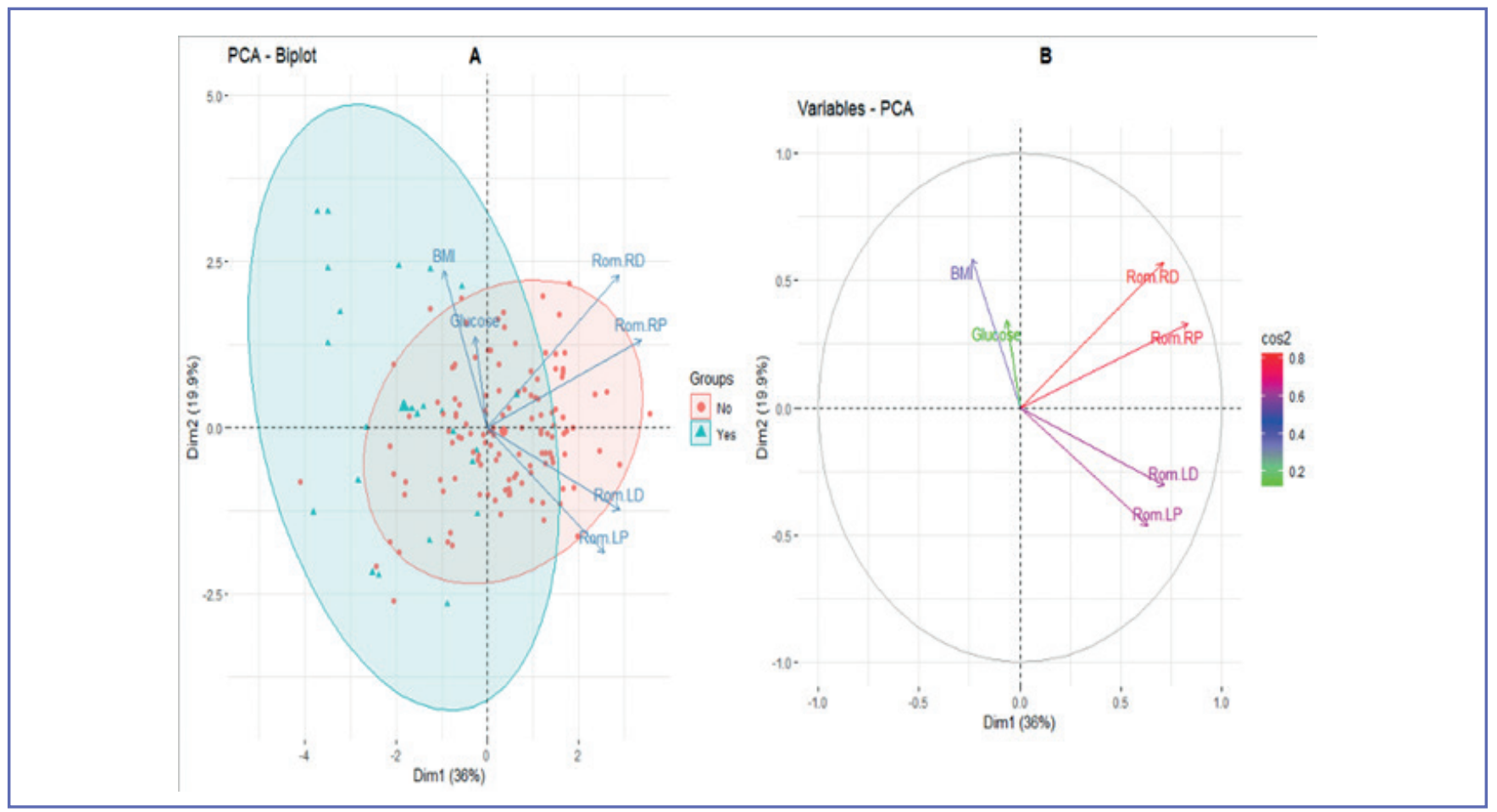

Figure 5. (A) Biplot of Principal Component Analysis (PCA). (B) Factor Map of Variables. The quality of representation of Blood Glucose is low. BMI, Rom LD, and Rom LP provided moderate representation and Rom RD and Rom RP exhibited high quality goodness of fit in Achilles tendinopathy. 
There are conflicting reports from prior studies on BMI as a significant risk factor for AT and most of the cohort studies found no significant association while a few linked obesity, and overweight with higher risks of developing AT (27, 28). Our findings reported a significant association between body mass index (BMI) and Achilles tendinopathy which agrees with previous findings even as the principal component analysis (PCA) also showed that the difference in BMI between participants with and without AT was statistically substantial $(\mathrm{p}=0.013)$.

One study in the review by Franceschi et al. (29) identified a potential interaction between age and obesity with degenerative tendon changes. Those with dyslipidemia and fat deposition in the Achilles tendon may be at risk for developing tendon pain. Another systematic review found that elevated adiposity was frequently associated with general tendon injuries (30). It was however noted from our findings that majority of the participants with Achilles tendinopathy had normal BMI $(16 ; 67 \%)$ so the underlying factor responsible for the significance appears to be beyond the body weight. It has been shown from prior studies that tendon disease is also prevalent in individuals with increased fat mass and greater waist/hip ratio despite having normal body weight. These group of individuals categorized as metabolically obese but of normal weight account for about 5 to $45 \%$ of the population $(24,31)$. This underscores the role of central obesity in tendinopathy and calls for further studies to investigate body composition as risk factor for AT.

Prior studies had reported restricted ankle motion, especially dorsiflexion in professional footballers $(5,32)$. This has been attributed to structural adaptations and was thought to be protective against injury by reducing excessive movements to which the joint is subjected. However, as far back as 1982, (33) it had been suggested that stiffness of the ankle joint in soccer players is a predisposing factor for muscle rupture and tendinitis which agrees with the findings of our study as $54 \%$ of the participants with AT had reduced Ankle dorsiflexion while $42 \%$ had reduced Ankle plantarflexion in the dominant limbs. For the left lower limbs, all the participants, even those without AT were seen to have both dorsiflexion and plantarflexion stiffness. As seen in figure 4, all the profiles of Ankle kinematics were significantly (p $<0.05$ ) associated with Achilles tendinopathy. However, logistic regression analysis identified right ankle dorsiflexion, left ankle plantarflexion and BMI as being significantly $(\mathrm{p}<0.05)$ associated with Achilles tendinopathy (table $\mathbf{V})$. These findings corroborate the report of structural adaptation of the soft tissues resulting in stiffness and underscores the need for preventive ankle stretching/flexibility exercises for football players even as some studies also reported that restricted ROM scores are primary risk factors for some of the most common football injuries $(34,35)$.

Diabetes has been identified as a major intrinsic factor for the development of Achilles tendinopathy even as recent systematic reviews have clearly demonstrated that patients with high cholesterol and diabetes are at significantly higher risk of developing tendinopathy (36). In our study, we found no association between Achilles tendinopathy and the blood glucose levels which is not consistent with the findings of the studies by Jarvinen et al. (37), and Bondi et al. (38) who reported that intrinsic risk factors for the development of Achilles tendinopathy included systemic diseases such as diabetes mellitus and elevated BMI. However, in the PCA factor map of variables (figure $\mathbf{5} \mathbf{~ B}$ ), it is seen that the quality of representation of blood glucose is low which implies that though it had no significant association in this study, it is still a factor to consider as a risk factor for AT. A prior study had actually advocated further investigations into the influence of hormones on tendon structure and metabolism (39). In addition, it is seen from our results that all the participants $(100 \%)$ with Achilles tendinopathy had normal glucose profile. This could be due to the role of genetic and racial factors as prior reports were from studies conducted among Caucasians.

Table V. Association between Achilles Tendinopathy and Selected Variables Using Logistic Regression.

\begin{tabular}{llccc}
\hline Parameters & Beta & Odds Ratio (OR) & 95\% OR & P value \\
\hline Age & 0.0004 & 1.0001 & $1-10.0012$ & 0.9937 \\
\hline BMI & 0.2548 & 1.2902 & $0.78-2.13$ & $0.024 * *$ \\
\hline Blood Glucose & -0.1505 & 0.8603 & $0.64-1.16$ & 0.7464 \\
\hline Rom RD & -0.002 & 0.9980 & $0.99-1.002$ & 0.999 \\
\hline Rom RP & -0.3086 & 0.7345 & $0.40-1.35$ & $0.008 * *$ \\
\hline Rom LD & -0.3826 & 0.6821 & $0.32-1.44$ & $0.038^{* *}$ \\
\hline Rom LP & -0.0491 & 0.9521 & $0.87-1.05$ & 0.688 \\
\hline
\end{tabular}

ROM - Range of Motion, RD - Right Dorsiflexion, RP - Right Plantarflexion, LD - Left Dorsiflexion, LP - Left Plantarflexion. 


\section{Limitations of Study}

This was a preliminary study and so the sample size was limited, a much larger sample size would have allowed for a more generalized application of the findings. Also, the role of body composition in Achilles tendinopathy was not determined. Further studies with a larger sample size and to determine the roles of body composition and genetic factors are advocated.

\section{CONCLUSIONS}

It was concluded that elevated body mass index and restricted ankle joint mobility were significant predisposing risk factors for Achilles tendinopathy among Nigerian footballers.

\section{CONFLICT OF INTERESTS}

The authors declare that the have no conflict of interests.

\section{REFERENCES}

1. Obst SJ, Barrett RS, Newsham-West R. Immediate effect of exercise on achilles tendon properties: systematic review. Med and Sci Sports and Exer 2013;45:1534-1544.

2. Owoeye OBA, Aiyegbusi AI, Fapojuwo OA, Badru OA, Babalola AR. Injuries in male and female semi-professional football (soccer) players in Nigeria: prospective study of a National Tournament. BMC Research Notes 2017;10:133.

3. Aiyegbusi AI, Okafor UAC, Leke OP. Prevalence of Achilles tendinopathy and its association with physical characteristics in recreational sport participants in Lagos, Nigeria. JCS 2016;13:163-6.

4. Hong-Yun L, Ying-Hui H. Achilles Tendinopathy: Current Concepts about the Basic Science and Clinical Treatments. BioMed Res Intern Article 2016;ID 6492597, 9 pages.

5. López-Valenciano A, Ayala F, Vera-García FJ, et al. Comprehensive profile of hip, knee and ankle ranges of motion in professional football players. J Sports Med Phys Fitness 2019;59(1):102-109.

6. Uquillas CA, Guss MS, Ryan DJ, Jazrawi LM, Strauss EJ. Everything Achilles: knowledge update and current concepts in management. AAOS exhibit selection. J Bone \& Joint Surg (Am) 2015;97(14):1187-1195.

7. Adae O. Amoako, Ariel N, Cory K. Body Mass Index as a Predictor of Injuries in Athletics. Current Sports Medicine Reports by the American College of Sports Medicine 2017;16(4):256-262.

8. Heyward OW, Rabello LM, van der Woude L, et al. The effect of load on Achilles tendon structure in novice runners. J Sci Med Sport 2018;21(7):661-665.

9. Saunders CJ, Collins M, September A, Schwellnus M. Extracellular matrix gene sequence variant analyses and Achilles tendinopathy. University of Cape Town, Published Thesis 2013. Assessed on 22/02/2019.
10. Whitting JW, Steele JR, McGhee DE, Munro BJ. Dorsiflexion capacity affects achilles tendon loading during drop landings. Med Sci in Sports and Ex 2011;43(4):706-713.

11. Mosler AB, Crossley KM, Thorborg K, Whiteley RJ, Weir A, Serner A. Hip strength and range of motion: normal values from a professional football league. J Sci Med Sport 2017;20(4):339-43.

12. Mason-Mackay AR, Whatman C, Reid D. The effect of reduced ankle dorsiflexion on lower extremity mechanics during landing: A systematic review. J Sci Med Sport 2017;20(5):451-458.

13. Arroyo F, Benardot D, Hernandez E. Within-Day Energy Balance in Mexican Female Soccer (Football) Players - An Exploratory Investigation. Inter J Sports and Ex Med 2018;4(4):104.

14. Hasan T, Ganesh K. Genetic regulators of Achilles tendon pathogenesis and outcomes. Global Adv Res J of Med and Medic Sci 2018;7(10):207-213.

15. Docking SI, Rio E, Cook J, Orchard J, Fortington LV. The prevalence of Achilles and patellar tendon injuries in Australian Football players beyond a time-loss definition. Scand J Med Sci Sports 2018;28(9):10.1111/sms.13086.

16. Padulo J, Frizziero A, Maffulli N. Basic Principles and Recommendation in Clinical and Field Science Research: 2018 update. MLTJ 2018;8(3):305-307.

17. Maffulli N, Kenward MG, Testa V, Capasso G, Regine R, King JB. Clinical diagnosis of Achilles tendinopathy with tendinosis. Clin J Sport Med 2003;13(1):11-5.

18. Nevin TW, James DC, Justin CL. Imaging in the Assessment and Management of Achilles Tendinopathy and Paratendinitis. Sem in MSK Radiol 2011;15:89-100.

19. Kibler W, McQueen C, Uhl T. Fitness Evaluations and Fitness Findings in competetive junior tennis players. Clin Sports Med 1988;7(2):403-16.

20. Kaiser, H.F. The Application of Electronic Computers to Factor Analysis. Educ. Psychol Meas 2020;20:141-151.

21. Zurutuza U, Castellano J, Echeazarra I, Guridi I, Casamichana D. Selecting Training-Load Measures to Explain Variability in Football Training Games. Front Psychol 2020;10:2897.

22. R Core Team. R: A language and environment for Statistical Computing. R Foundation for Statistical Computing, Vienna, Austria, 2020.

23. Lewis JS. Rotator cuff tendinopathy. $\mathrm{Br} \mathrm{J}$ Sports Med 2009;43(4):236-41.

24. Gaida JE, Alfredson H, Kiss ZS, Bass SL, Cook JL. Asymptomatic Achilles tendon pathology is associated with a central fat distribution in men and a peripheral fat distribution in women: a cross sectional study of 298 individuals. BMC Musculoskelet Disord 2010;11:41.

25. Bouchard C, Després JP, Mauriège P. Genetic and nongenetic determinants of regional fat distribution. Endocr Rev 1993;14(1):72-93

26. Scott A, Zwerver J, Grewal N, de Sa A, Alktebi T, Granville DJ, Hart DA. Lipids, adiposity and tendinopathy: is there a mechanistic link? Critical review. Br J Sports Med 2015;49(15):984-8.

27. Wezenbeek E, Willems T, Mahieu N, et al. Role of the Vascular and Structural Response to Activity in the Development of Achilles Tendinopathy: A Prospective Study. Am J Sports Med 2018;46(4):947-954.

28. Abate M, Oliva F, Schiavone C, Salini V. Achilles tendinopathy in amateur runners: role of adiposity (Tendinopathies and obesity). MLTJ 2012;2(1):44-48. 
29. Franceschi F, Papalia R, Paciotti M, et al. Obesity as a Risk Factor for Tendinopathy: A Systematic Review. Intern J Endocr 2014;ID 670262.

30. Gaida JE, Ashe MC, Bass SL. Cook JL. Is adiposity another recognized risk factor for tendinopathy? A systematic review. Arthritis Care and Res 2009;61(6):840-849.

31. Conus F, Rabasa-Lhoret R, Péronnet F. Characteristics of metabolically obese normal-weight (MONW) subjects. Appl Physiol Nutr Metab 2007;32:4-12.

32. Martin RL, Chimenti R, Cuddeford T, et al. Achilles Pain, Stiffness, and Muscle Power Deficits: Midportion Achilles Tendinopathy Revision. J Orthop Sports Phy Therapy 2018;48(5):A1-A38.

33. Hattori K, Ohta S. Ankle Joint Flexibility in College Soccer Players. J Human Ergol 1986;15:85-89.

34. Fousekis K, Tsepis E, Poulmedis P, Athanasopoulos S, Vagenas $G$. Intrinsic risk factors of non-contact quadriceps and hamstring strains in soccer: a prospective study of 100 professional players. Br J Sports Med 2011;45(9):709-14.

35. Daneshjoo A, Rahnama N, Mokhtar AH, Yusof A. Bilateral and unilateral asymmetries of isokinetic strength and flexibility in male young professional soccer players. J Hum Kinetics 2013;36(1):45-53.

36. Ranger TA, Wong AM, Cook JL, Gaida JE. Is there an association between tendinopathy and diabetes mellitus? A systematic review with meta-analysis. Br J Sports Med 2016;50(16):982-989.

37. Järvinen TAH, Kannus P, Maffulli N. Achilles tendon disorders: etiology and epidemiology. Foot Ank Clin J 2005;10(2):255-266.

38. Bondi M, Rossi N, Magnan B, Brivio LR. The Achilles Tendinopathy: Pathogenesis Review. Intern J Orthop 2(3):289-299.

39. Oliva F, Piccirilli E, Berardi AC, Frizziero A, Tarantino U, Maffulli N. Hormones and tendinopathies: the current evidence. Br Med Bull 2016;117(1):39-58. 